\title{
Unexpected Etiology in a Case of Bilateral Maculopathy
}

\author{
Prem Patel $^{a} \quad$ Kaylie Jones $^{b}$ Deborah I. Friedman ${ }^{a, c}$ David G. Birch ${ }^{b}$ \\ Rafael L. Ufret-Vincenty ${ }^{a}$ \\ aDepartment of Ophthalmology, UT Southwestern Medical Center, Dallas, TX, USA; betina \\ Foundation of the Southwest, Dallas, TX, USA; 'Department of Neurology, UT Southwestern \\ Medical Center, Dallas, TX, USA
}

\section{Keywords}

Bilateral maculopathy · Hydroxychloroquine · Plaquenil · Genetic mutation · HGSNAT

\begin{abstract}
A 74-year-old woman with a history of rheumatoid arthritis using hydroxychloroquine presented with gradually progressive decreased vision in both eyes and was found to have a bilateral maculopathy. Initial genetic testing was negative, and after discussing the low likelihood of her severe findings being secondary to her relatively low hydroxychloroquine exposure, the possibility of an autoimmune retinopathy was entertained. Updated data on the genetic testing reclassified one of her mutations in HGSNAT as pathogenic. This case highlights the value of genetic testing and the need to keep a high index of suspicion even after initial negative results, given the fact that our knowledge of mutations leading to retinal degeneration is constantly evolving.
\end{abstract}

\section{Introduction}

The differential diagnosis of bilateral maculopathies includes a wide range of etiologies including age-related macular degeneration, myopic degeneration, autoimmune or cancerassociated retinopathy, toxicity, and genetic disorders. Although it is clearly important to establish an accurate diagnosis, this can be challenging due to the nonspecific nature of the findings, potential interplay of these factors, and our limited knowledge of specific mutations that lead to maculopathy. Here, we report the diagnosis and workup of an atypical case, which combined a bilateral maculopathy with mild optic nerve and vascular changes, in a patient

\section{Karger!}


Patel et al.: Unexpected Cause of Maculopathy

with exposure to hydroxychloroquine who ended up harboring a homozygous HGSNAT mutation.

\section{Case Presentation}

A 74-year-old woman with a history of rheumatoid arthritis was referred due to a 2-year history of painless, progressive, bilateral decline in vision, particularly pericentral vision. She also had difficulty seeing in the dark. There was no family history of retinal disorders. She took $200 \mathrm{mg} /$ day of hydroxychloroquine for two and a half years to treat her rheumatoid arthritis (started about 6 months to 1 year before her symptoms began). She had seen several ophthalmologists at the time of presentation, and an MRI of the brain had been obtained, which showed mild bilateral white matter changes but no signs of mass lesion, optic nerve compression, or multiple sclerosis that could explain her vision loss.

Examination showed visual acuity of 20/40 in both eyes. Farnsworth D15 color testing was normal. There was mild optic disc pallor and some arteriolar attenuation in both eyes (Fig. 1a, b). At least one arteriole appeared attenuated/occluded but without vasculitis sheathing (white arrows in Fig. 1a). The most prominent aspect of her presentation was pigmentary changes/atrophy in both eyes in a circular pattern involving the macula and entire posterior pole (Fig. 1a, b). This pattern was quite pronounced on fluorescein angiography (Fig. 1c, d). Goldmann perimetry showed a significant depression of the hill of vision, but the larger targets (V4e and III4e) showed normal contour and minimal constriction (Fig. 1e, f). OCT images showed loss of outer retinal layers involving the entire macula, except for the fovea in both eyes (Fig. 1g-j). Thus, although not isolated, a bilateral maculopathy was the primary component of the clinical presentation. The full-field electroretinography (ffERG; Fig. 2) had borderline normal amplitudes for her age. Multifocal ERG was difficult because the patient had very unstable fixation but showed low/noisy signals. There was no evidence of vasculitis on angiography.

Our differential diagnosis included hydroxychloroquine toxicity, genetic causes, cancer-associated retinopathy, or autoimmune retinopathy. Although severe cases of hydroxychloroquine toxicity could potentially lead to outer retinal changes, disc pallor, and perhaps even vascular attenuation, this usually occurs with much higher doses of hydroxychloroquine than the $5 \mathrm{mg} / \mathrm{kg} /$ day dosage that our patient took for 2.5 years. She had no kidney disease and never used tamoxifen. Genetic testing for retinal dystrophies was obtained at the Retina Foundation of the Southwest, but no relevant mutations were found. We discussed testing for antiretinal antibodies, but the patient deferred. She agreed to a systemic screening for malignancy, which was negative. Although the progression of visual loss in autoimmune retinopathy tends to be more rapid than what she had experienced and ffERG typically shows more pronounced changes, given the exclusion of other potential etiologies, our patient was insisted on trying empirical therapy for autoimmune retinopathy for 1 year with methotrexate (10 mg/week orally) and was proceeded with treatment after a careful discussion about potential risks with a rheumatologist. At the end of the year, she experienced persistent deterioration in vision, and evidence of progression was clearly seen on fundus autofluorescence (Fig. 3). During the process of discussing alternatives, we received notification that a previously identified variant in her genetic test affecting HGSNAT had just been reclassified as pathogenic. The patient was found to be homozygous for the missense variant HGSNAT c.1843G > A, p.(Ala615Thr), which was previously considered benign due to its high allele frequency in control populations (1116/273822, Genome Aggregation Database). New evidence indicated that this variant functions as a mild hypomorphic allele and was reported in patients with nonsyndromic retinitis pigmentosa $[1,2]$.

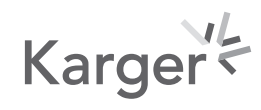




\section{Case Reports in Ophthalmology}

\begin{tabular}{l|l}
\hline Case Rep Ophthalmol 2021;12:622-628 \\
\hline DOI: 10.1159/000514705 & $\begin{array}{l}\text { ○ 2021 The Author(s). Published by S. Karger AG, Basel } \\
\text { www.karger.com/cop }\end{array}$ \\
\hline
\end{tabular}

Patel et al.: Unexpected Cause of Maculopathy
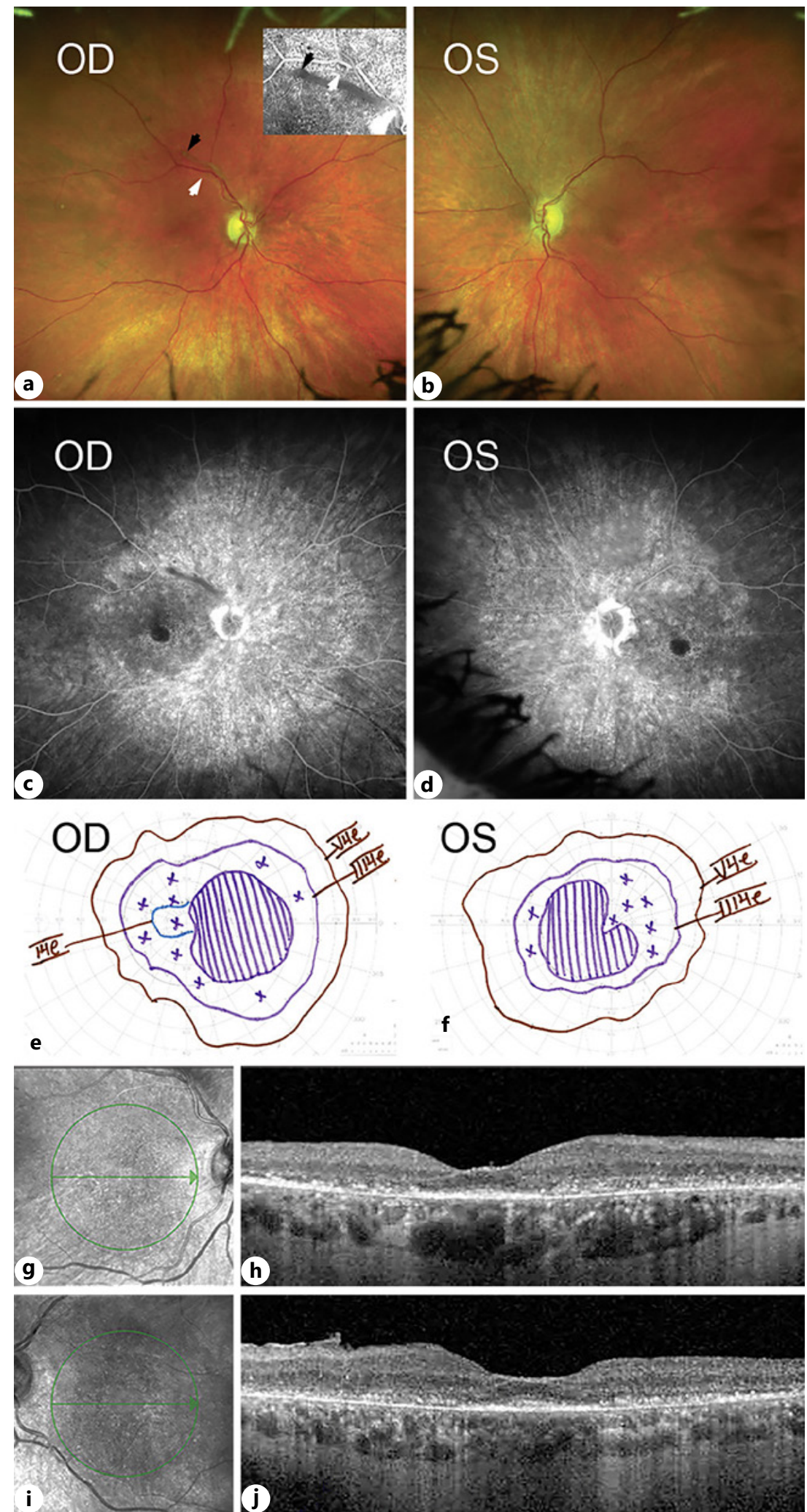

Fig. 1. Fundus photographs (a, b), late fluorescein angiogram (c, d), and Goldmann visual field (e, f) at presentation. a An inset with a mid-phase angiogram to point to an attenuated/occluded arteriole (white arrows) and a moving vitreous condensation (black arrows). $\mathbf{g}-\mathbf{j}$ Infrared and OCT images at presentation are also shown. 
Fig. 2. ERG showing rod (a), combined (b), and cone flicker (c) signals within normal limits for age. ffERG recordings were obtained using the ISCEV) standard protocol. ffERG, full-field electroretinogram; ISCEV, International Society for Clinical Electrophysiology of Vision.

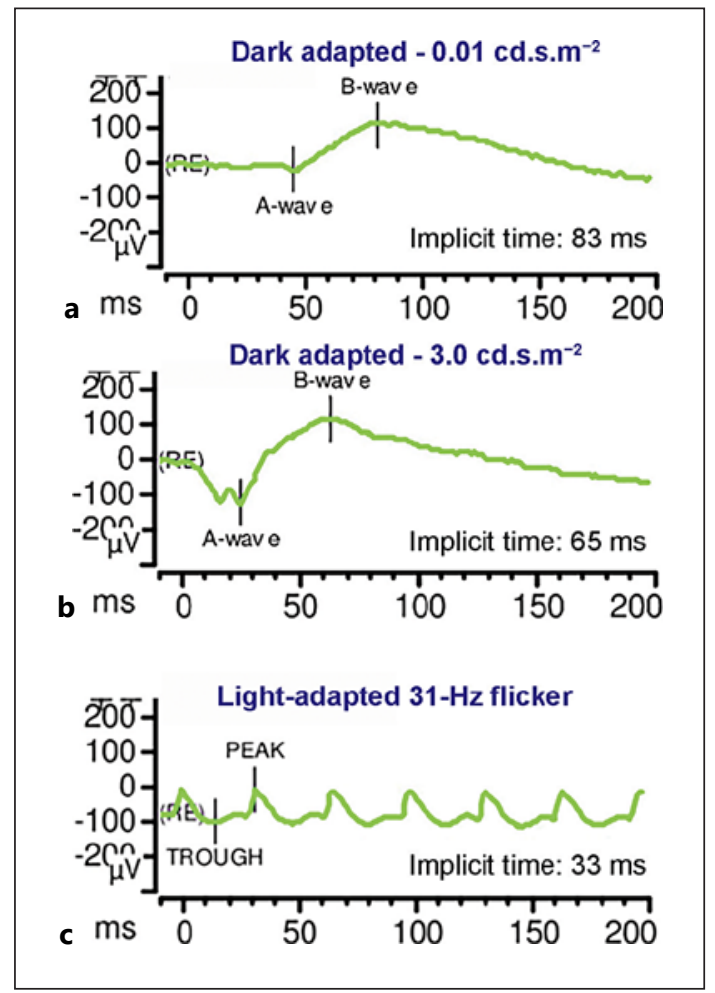

\section{Discussion}

Mutations in HGSNAT are classically tied to mucopolysaccharidosis type IIIC (MPS IIIC or Sanfilippo type C syndrome) and recently associated with nonsyndromic RP and pericentral RP. Our patient's presentation of nyctalopia and loss of peripheral vision and the lack of other organ or sensory system involvement supported a diagnosis of either nonsyndromic RP [1] or pericentral RP [2]. Disc pallor, as observed in our patient, has been reported in patients diagnosed with nonsyndromic RP [2]. Haer-Wigman et al. [1] found that the HGSNAT mutations leading to nonsyndromic RP were associated with levels of HGSNAT that decreased compared to healthy controls but were higher than those in patients with MPS IIIC. They proposed that the retina was more dependent on HGSNAT than the brain or other tissues, and thus, only the retina was affected in the patients they reported. One of the families they identified had 2 variants in the HGSNAT gene (c.398G > C and c.1843G > A), and the 3 individuals with retinal degeneration in the family had the onset of symptoms at 47-52 years of age. The other individuals in their report had a different mutation in HGSNAT (c.370A > T) and presented with symptoms in childhood. Comander et al. [2] identified other HGSNAT mutations leading to nonsyndromic pericentral RP. The constellation of retinal findings was again somewhat different from those reported by Haer-Wigman et al. [1] and in our patient, with some patients showing bone spicules and some only outer retinal atrophy. However, a consistent finding in all of the reported cases and ours is that the pericentral retina is more affected than the fovea or the far retinal periphery. In our case, this pericentral distribution was underscored by (1) the imaging, (2) the fairly preserved ffERG signals, and (3) affected HVF and diminished vision hill in Goldmann perimetry with fairly preserved III4e and V4e isopters. Several mutations may produce this pericentral pattern of retinal degeneration, including PRPF31, RHO, HK1, and USH2A [3]. In contrast to previously described cases, our patient's symptoms started much later, around age 70 years, and her ocular findings were 

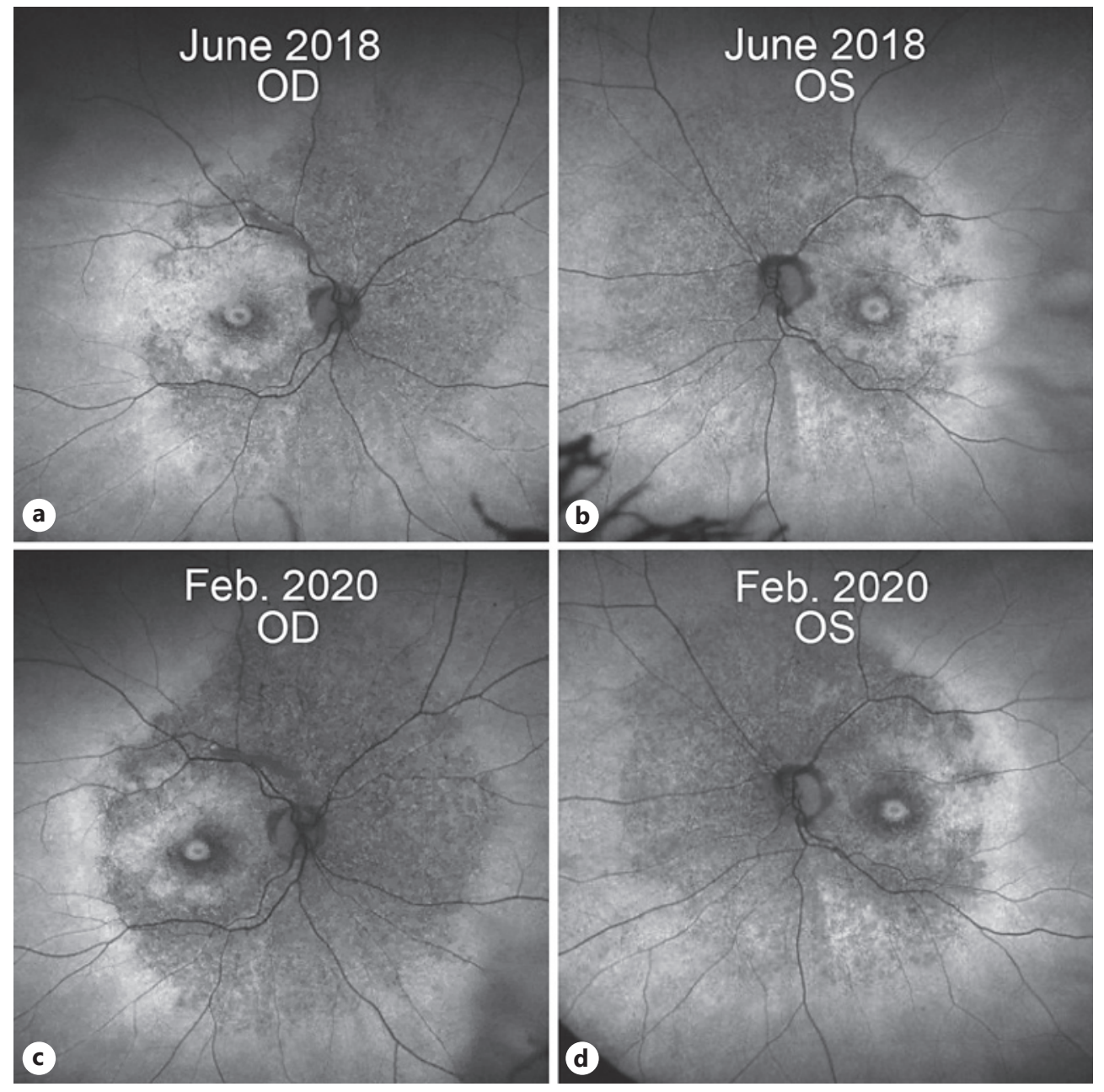

Fig. 3. Fundus autofluorescence in 2018 (a, b) and 2020 (c, d).

somewhat different from those reported by others describing these rare cases of HGSNATrelated degenerations. She had a combination of optic nerve pallor, vascular attenuation, absence of bone spicules, and presence of outer retinal atrophy involving the entire posterior pole, except for the fovea. Her mutation (c.1843G > A) was one of the 2 HGSNAT mutations occurring simultaneously in one of the families reported by Haer-Wigman et al. [1]. Thus, the phenotype may be partly dependent on the exact mutation(s) and their effect on the levels of protein expression and perhaps protein configuration/function.

Another interesting aspect of our case is the possibility that hydroxychloroquine use may have played a contributing role. The patient was initially referred due to a concern for hydroxychloroquine toxicity. Although hydroxychloroquine has been reported to cause toxicity even after short-term use [4], this is very rare. The estimated risk of toxicity in the first 5 years of use is only 1\% [5]. Given that our patient took a low dose of hydroxychoroquine, toxicity is highly unlikely after a 2.5-year course of treatment. The history of progression of maculopathy after discontinuation of hydroxychloroquine would not rule out a toxic etiology, since it is well-documented that progression can occur years after discontinuation of the drug [6]. Of course, there is high biologic variability, and some patients are much more susceptible to macular toxicity. In fact, some patients develop severe toxicity with arteriolar narrowing and disc pallor [7]. However, these findings are rare, even in patients with long 
exposure to high doses of hydroxychloroquine. These inconsistencies made it unlikely that hydroxychloroquine alone explained our patient's findings. However, it is still possible that hydroxychloroquine could have contributed to the onset and severity of the presentation. A case of a compound effect of hydroxychloroquine toxicity and a heterozygous mutation in USH2A has been reported [8]. Furthermore, patients with recessive Stargardt disease (homozygous $A B C A 4$ mutations) can develop maculopathy that is very similar to that seen in plaquenil toxicity [9]. Finally, a study found evidence for an increased predisposition to HCQ toxicity in patients with $A B C A 4$ mutations [10].

\section{Conclusion}

Our case not only illustrates the diagnostic challenges in patients with retinal dystrophies but the fact that our knowledge of pathogenic mutations is still evolving. New pathogenic mutations are still being identified. Furthermore, even for mutations known to be relevant to retinal development or homeostasis, we are still learning about variations in the resulting clinical phenotypes. The recent reclassification of the patient's HGSNAT mutation is a case in point.

\section{Statement of Ethics}

While there is no personally identifiable information in this report, written consent for publication was still obtained from the patient.

\section{Conflict of Interest Statement}

There are no conflicts of interest to report.

\section{Funding Sources}

This study was supported by an unrestricted grant from Research to Prevent Blindness.

\section{Author Contributions}

Prem Patel: original draft preparation, literature search, and manuscript writing/ drafting. Kaylie Jones, MS and David Birch, PhD: data interpretation and analysis, critical review of the manuscript, and manuscript editing and revision. Deborah Friedman: critically reviewed and edited the manuscript. Rafael Ufret-Vincenty, MD: design, data acquisition and interpretation, manuscript drafting and editing, amd literature search. All the authors read and approved the final manuscript.

\section{References}

1 Haer-Wigman L, Newman H, Leibu R, Bax NM, Baris HN, Rizel L, et al. Non-syndromic retinitis pigmentosa due to mutations in the mucopolysaccharidosis type IIIC gene, heparan-alpha-glucosaminide $\mathrm{N}$-acetyltransferase (HGSNAT). Hum Mol Genet. 2015;24(13):3742-51.

\section{Karger'}


Patel et al.: Unexpected Cause of Maculopathy

2 Comander J, Weigel-DiFranco C, Maher M, Place E, Wan A, Harper S, et al. The genetic basis of pericentral retinitis pigmentosa-a form of mild retinitis pigmentosa. Genes. 2017;8(10):256.

3 Matsui R, Cideciyan AV, Schwartz SB, Sumaroka A, Roman AJ, Swider M, et al. Molecular heterogeneity within the clinical diagnosis of pericentral retinal degeneration. Invest Ophthalmol Vis Sci. 2015;56(10):6007-18.

4 Pasaoglu I, Onmez FE. Macular toxicity after short-term hydroxychloroquine therapy. Indian J Ophthalmol. 2019;67(2):289-92.

5 Marmor MF, Kellner U, Lai TY, Melles RB, Mieler WF. American academy of ophthalmology. Recommendations on screening for chloroquine and hydroxychloroquine retinopathy (2016 revision). Ophthalmology. 2016; 123(6):1386-94.

6 Mititelu M, Wong BJ, Brenner M, Bryar PJ, Jampol LM, Fawzi AA. Progression of hydroxychloroquine toxic effects after drug therapy cessation: new evidence from multimodal imaging. JAMA Ophthalmol. 2013;131(9): 1187-97.

7 Stokkermans TJ, Goyal A, Bansal P, Trichonas G. Chloroquine and hydroxychloroquine toxicity. In: StatPearls. Treasure Island, FL: StatPearls Publishing; 2020 Jan. Available from: https://www.ncbi.nlm.nih.gov/books/ NBK537086/.

8 Katsman D, Sanfilippo C, Sarraf D. Panretinal degeneration associated with long-term hydroxychloroquine use and heterozygous USH2A mutation. Retin Cases Brief Rep. 2017;11(Suppl 1):S77-80.

9 Nõupuu K, Lee W, Zernant J, Greenstein VC, Tsang S, Allikmets R. Recessive Stargardt disease phenocopying hydroxychloroquine retinopathy. Graefes Arch Clin Exp Ophthalmol. 2016;254:865-72.

10 Shroyer NF, Lewis RA, Lupski JR. Analysis of the ABCR (ABCA4) gene in 4-aminoquinoline retinopathy: is retinal toxicity by chloroquine and hydroxychloroquine related to Stargardt disease? Am J Ophthalmol. 2001; 131:761-6. 